\title{
Early oral colostrum administration in preterm infants
}

\author{
Diana Maffei ${ }^{1} \cdot$ Mariana Brewer $^{1} \cdot$ Champa Codipilly $^{1} \cdot$ Barry Weinberger $^{1} \cdot$ Richard J. Schanler $^{1}$
}

Received: 26 April 2019 / Revised: 14 October 2019 / Accepted: 7 November 2019 / Published online: 20 November 2019

(c) The Author(s), under exclusive licence to Springer Nature America, Inc. 2019

\begin{abstract}
Background Early administration of colostrum may provide preterm infants with immune components. Previous studies illustrating the effects of oral colostrum (OC) have been confounded by the coincidence of enteral feedings.

Objective To quantify OC absorption, as measured by urinary $\operatorname{sg} \mathrm{A}$ and lactoferrin, in preterm infants prior to enteral feedings.

Materials and methods Colostrum was obtained from mothers delivering infants $\leq 32$ weeks and $\leq 1500 \mathrm{~g}$. $\operatorname{sg} \mathrm{A}$ and lactoferrin were measured in infant urine, and microflora in saliva and tracheal aspirates were characterized.

Results Urinary sIgA and lactoferrin were significantly greater in infants receiving OC by syringe compared to swab $(p<$ 0.002). Urinary sIgA correlated with the total number of doses in $72 \mathrm{~h}\left(R^{2}=43 \%, p<0.01\right)$.

Conclusions Administration of OC by syringe and higher cumulative dose are associated with increased absorption of $\operatorname{sig} \mathrm{A}$ and lactoferrin, and early dosing may contribute to a more diverse tracheal microbiome.
\end{abstract}

\section{Introduction}

Colostrum from mothers of preterm infants contains high concentrations of immune factors [1-3]. Early exposure to colostrum, however, is limited not only due to maternal reasons but because preterm infants often have delayed feedings and small quantities of milk may be difficult to administer with intragastric tube feedings [4-6]. Application of colostrum to the buccal mucosa (oral colostrum, OC) may provide early immunologic benefit to the preterm infant and provision of colostrum may facilitate maternal milk production [7].

Although a previous investigation reported absorption of human milk proteins, $\operatorname{sIg} \mathrm{A}$ and lactoferrin, as a function of OC exposure, coincident intragastric tube feedings of human milk may have contributed to the outcomes [8]. Additional investigations suggested that human milk as the first feeding and the feeding at discharge were more likely after receipt of OC $[8,9]$. OC also may impact the oral microbiome by stimulating lymphoid tissue and modulating bacterial adhesion

Diana Maffei
dmaffei@northwell.edu

1 Division of Neonatal-Perinatal Medicine, Cohen Children's Medical Center, Lilling Family Neonatal Research Lab, Feinstein Institutes for Medical Research, Zucker School of Medicine at Hofstra/Northwell, New Hyde Park, Hempstead, NY, USA
$[10,11]$. It has been suggested that OC alters bacterial colonization in the trachea of intubated infants and decreases ventilator-associated airway infections [10].

Despite the apparent safety and feasibility of OC exposure, there are no standards for the collection of milk, transfer of milk to the infant, and frequency and method of administration $[6,9,10]$. The aims of this pilot study were to document the use of $\mathrm{OC}$ in a preterm infant population, determine whether selected human milk immune proteins are absorbed following $\mathrm{OC}$ exposure in absence of feeding, and to characterize the effects of $\mathrm{OC}$ on the oral and tracheal microflora.

\section{Materials and methods}

Preterm infants ( $\leq 32$ weeks gestation and $\leq 1500 \mathrm{~g}$ birth weight) who did not receive enteral feedings for $72 \mathrm{~h}$ were eligible for recruitment and enrolled from February 2016 to December 2017. Study personnel reviewed the timing and dosing of OC prior to $72 \mathrm{~h}$, collected urine samples at $72 \mathrm{~h}$, and, if infants were intubated collected samples of tracheal secretions at $72 \mathrm{~h}$ and 7 days. The study was approved by the Northwell Health Institutional Review Board, and informed consent was obtained from parents. Infants with major congenital abnormalities or those expected not to survive $>1$ week were not enrolled. Maternal decision for breastfeeding was not a requirement for enrollment, but $90 \%$ of mothers eventually expressed their milk. 


\section{OC administration}

The routine care of infants in the neonatal intensive care unit (NICU) included lactation consultants working with mothers to ensure early milk expression and provision of OC to infants as soon as it is available, with no specific contraindications to its use. Labor \& Delivery personnel were asked to discuss manual milk expression with mothers. NICU staff were instructed on administration of OC to the buccal mucosa bilaterally every $3 \mathrm{~h}$ as available. Initially, cotton swabs were used, but in October 2016 this practice was changed to the use of tuberculin syringes to deliver $0.1 \mathrm{~mL}$ to each buccal surface. The number and frequency of doses were counted.

\section{Determination of urinary secretory $\lg A$ and lactoferrin}

Urine was collected at $72 \mathrm{~h}$ after birth, before any enteral feedings were initiated, using a sterile collection bag in the diaper. Samples were aliquoted and stored at $-80^{\circ} \mathrm{C}$ until analyzed. Secretory IgA (ABCAM, Cambridge, MA) and lactoferrin (Eagle Biosciences, Nashua, NH) were analyzed by enzyme-linked immunosorbent assay. Urinary creatinine (Cayman Chemical, Ann Arbor, Michigan) was used to adjust for differences in urinary output.

\section{Microbiome analysis}

Samples of saliva were collected at $72 \mathrm{~h}$. Tracheal secretions were obtained via in-line suctioning at $72 \mathrm{~h}$ and day 7 if infants were intubated. DNA was isolated from saliva and tracheal samples using a ZymoBiomics DNA Mini Kit (Irvine, CA). Polymerase chain reaction (PCR) of the $16 \mathrm{~s}$ ribosomal DNA (16 s rDNA) was performed according to the methods of Tannock [12]. The PCR product was sequenced using the Illumina-curated version of the May 2013 release of the Greengene Consortium Database. Shannon Diversity Index (SDI) was calculated as a measure of genus diversity [13].

\section{Demographic and outcome data}

The following data were obtained from the electronic medical record: timing and frequency of OC administration in the first $72 \mathrm{~h}$; birth weight; gestational age; receipt of antibiotics or other medications; comorbidities including infection, necrotizing enterocolitis, bronchopulmonary dysplasia, retinopathy of prematurity; and timing and type of enteral nutrition. Maternal demographics, including receipt of antibiotics and/or chorioamnionitis, were recorded.

\section{Statistics}

As a pilot study, no power analysis or calculation of sample size was performed. Univariate and multivariable regression were used to determine relationships between OC dose (frequency) and urinary concentrations of $\operatorname{sIg} \mathrm{A}$ and lactoferrin. Univariable linear regression analyses were performed to examine the association between SDI and timing of initial OC and cumulative number of OC administrations. Analysis was conducted using SAS version 9.4 (SAS Institute, Inc., Cary, NC).

\section{Results}

The time to receipt of first $\mathrm{OC}$ was $32 \pm 22 \mathrm{~h}$ (mean $\pm \mathrm{SD}$, range 4-66h). OC was given to $15(31 \%)$ and $20(42 \%)$ infants by 24 and $36 \mathrm{~h}$, respectively. During the $72 \mathrm{~h}$ study interval, infants received a median of 7.5 doses (range 1-16). The number of OC doses received in the first $24 \mathrm{~h}$ was predictive of the total OC doses in $72 \mathrm{~h}, \mathrm{R}^{2}=32 \%, p<0.001$. Of 48 consecutive infants enrolled, 16 (33\%) did not receive OC by $72 \mathrm{~h}$. When comparing OC and no OC infants, there were no differences in birth weight (overall mean $895 \pm 266 \mathrm{~g}$ ), gestational age (overall mean $26.6 \pm 2.5$ weeks), and incidence of Cesarean delivery (overall $81 \%$ ).

OC was administered to the buccal mucosa by swab in $20(42 \%)$ infants and by syringe in $28(58 \%)$ infants. There were no differences between swab vs syringe administration for birth weight, gestational age, and number of colostrum doses. The method of OC administration was not associated with the number of OC doses by $72 \mathrm{~h}$ or the time to full feedings ( $25 \pm 15$ days).

Colostrum administration by syringe was associated with significantly greater urinary $\operatorname{sIgA}$ and lactoferrin concentrations than by swab (median $427 \mathrm{vs} 4 \mathrm{ng} / \mathrm{mL}$ and 5 vs $0.8 \mathrm{ng} / \mathrm{mL}$, respectively, Fig. 1). These findings were not affected by normalizing to creatinine as a marker of urine concentration. There was a direct relationship between urinary $\operatorname{SIgA}$ and the number of OC doses by $72 \mathrm{~h}, R^{2}=$ $43 \%, p=0.001$, Fig. 2 .

In this pilot study, we found no significant differences between cumulative doses of OC and distribution of bacterial genera, or bacterial diversity as quantified by SDI, in saliva at $72 \mathrm{~h}$. Similarly, there was no significant association between the time of first $\mathrm{OC}$ dose and the composition or SDI of the oral or tracheal microbiota at $72 \mathrm{~h}$. There was a trend toward decreased SDI in trachea on day 7 with increased time to first OC administration, $R^{2}=19 \%, p=$ 0.088. Method of OC administration was not associated with the incidence of sepsis (34\%), bronchopulmonary dysplasia (38\%), and necrotizing enterocolitis (6\%), but differences were observed between swab and syringe 


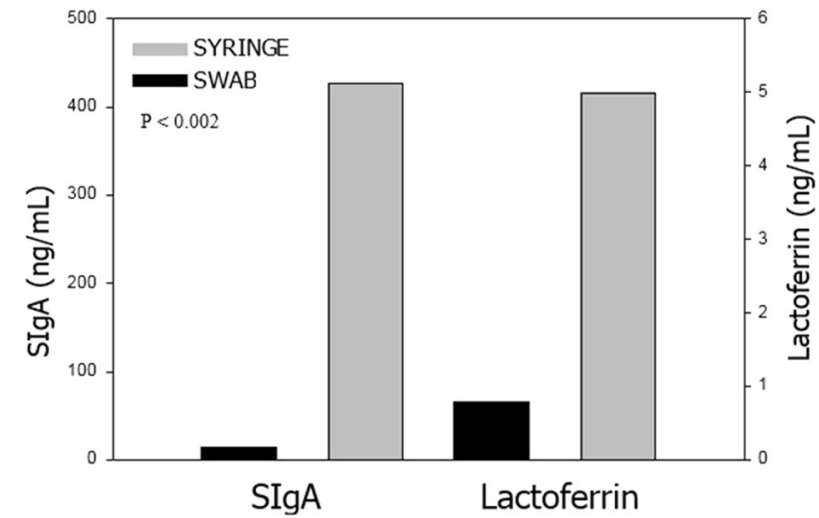

Fig. 1 Urine $\operatorname{sg} \mathrm{A}$ and lactoferrin concentrations after oral colostrum administered by swab or syringe

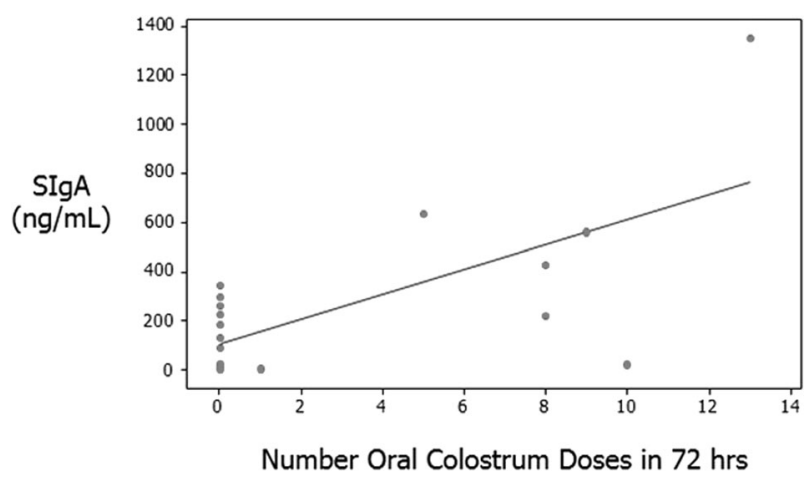

Fig. 2 Relationship between urine sIgA and colostrum doses received via syringe

administration for retinopathy of prematurity, $26 \%$ vs $0 \%$, $p=0.016$. Overall, infants with sepsis had less lactoferrin absorbed than those without sepsis, $1.2 \pm 1.4$ vs $4.3 \pm$ $3.7 \mathrm{ng} / \mathrm{mL}, p=0.025$.

\section{Discussion}

Although OC is emerging as a part of routine care for preterm infants, practices differ widely. Using urine concentrations as a marker for intestinal absorption of milk-derived immune proteins, we found that uptake of $\operatorname{sg} \mathrm{A}$ and lactoferrin were optimal when delivered to the buccal mucosa via tuberculin syringe rather than by cotton swab [14]. Of note, urinary $\operatorname{sg} A$ was positively associated with total number of OC doses received. This finding is novel because all sampling was done prior to the initiation of enteral feedings, so absorbed $\operatorname{sgA}$ and lactoferrin are attributable solely to intake via OC. Early and ongoing administration of $\mathrm{OC}$ by syringe is likely to optimize innate immune defenses in preterm infants exposed to a human milk diet.

It was concerning that, despite the emphasis on the importance of human milk and OC in our NICU, the median time to first $\mathrm{OC}$ administration was more than $24 \mathrm{~h}$ after delivery and less than half of the infants received $\mathrm{OC}$ in the first $36 \mathrm{~h}$. Since early OC results in greater cumulative doses administered and, therefore, greater immune protein concentrations, it is necessary to further emphasize the importance of early milk expression to all staff. These findings support the idea that mothers of preterm infants face unique challenges to early milk production, likely related either to insufficient breast priming, stress, or medical diagnoses that contributed to preterm labor. Although there was staff education, more direct support from staff in Labor \& Delivery and NICU is required. We now emphasize the importance of immediate postpartum milk expression during antenatal consultations for all mothers likely to deliver prematurely. We have empowered our staff to encourage transfer of any amount of milk to the NICU immediately after delivery.

OC did not significantly alter the composition of the oral and tracheal microflora. The trend toward decreased microbial diversity in the trachea with increased time to first OC suggests that early OC may contribute to development of a more robust and protective microbiome. The microbiome data may contribute to understanding the association between a human milk diet in preterm infants and protection from bronchopulmonary dysplasia [15].

Limitations of this study include the lack of randomization. The change from swab to syringe OC was adopted throughout the NICU and observations were not concurrent, so they may be confounded by other alterations in practice. As all recruitment was done within a 2 year period, this effect is unlikely to be significant. The differences in retinopathy of prematurity, however, reflect the practice changes in monitoring oxygen satuartions during this time period. This study enrolled infants who were not ordered to receive enteral feedings until after $72 \mathrm{~h}$. As this practice is contrary to our NICU guidelines of early enteral nutrition, it is likely that study infants were sicker than the usual preterm population, and provides a rationale for the observations of delays in time to full enteral feeding and the higher incidence of comorbidities.

In summary, we found that immune proteins in human milk are absorbed after early OC administration and that early colostrum is best administered onto the buccal mucosa by syringe. Greater cumulative OC doses are associated with increased absorption of $\operatorname{sIgA}$ and lactoferrin. The delays in receipt of OC support the need for further efforts to obtain milk as soon as possible after preterm delivery.

\section{Compliance with ethical standards}

Conflict of interest The authors declare that they have no conflict of interest. 
Publisher's note Springer Nature remains neutral with regard to jurisdictional claims in published maps and institutional affiliations.

\section{References}

1. Dvorak B, Fituch CC, Williams CS, Hurst NM, Schanler RJ. Increased epidermal growth factor levels in human milk of mothers with extremely premature infants. Pediatr Res. 2003;54:15-9.

2. Trend S, Strunk T, Lloyd ML, Kok CH, Metcalfe J, Geddes DT, et al. Levels of innate immune factors in preterm and term mothers' breast milk during the 1st month postpartum. Br J Nutr. 2016;115:1178-93.

3. Koenig A, Diniz EMA, Barbarosa SFC, Vaz FAC. Immunologic factors in human milk: the effects of gestational age and pasteurization. J Hum Lact. 2005;21:439-43.

4. Meier PP, Engstrom JL, Patel AL, Jegier BJ, Bruns NE. Improving the use of human milk during and after the NICU stay. Clin Perinatol. 2010;37:217-45.

5. Nasuf AWA, Ojha S, Dorling J. Oropharyngeal colostrum in preventing mortality and morbidity in preterm infants. Cochrane Database Syst Rev. 2018;9:CD011921.

6. Pletsch D, Ulrich C, Angelini M, Fernandes G, Lee DS. Mothers' "liquid gold": a quality improvement initiative to support early colostrum delivery via oral immune therapy (OIT) to premature and critically ill newborns. Nurs Leadersh. 2013;26:34-42.

7. Parker LA, Sullivan S, Krueger C, Kelechi T, Mueller M. Effect of early breast milk expression on milk volume and timing of lactogenesis stage II among mothers of very low birth weight infants: a pilot study. J Perinatol. 2012;32:1-5.

8. Lee J, Kim HS, Jung YH, Choi KY, Shin SH, Kim EK, et al. Oropharyngeal colostrum administration in extremely premature infants: an RCT. Pediatrics. 2015;135:e357-66.

9. Snyder R, Herdt A, Mejias-Cepeda N, Ladino J, Crowley K, Levy P. Early provision of oropharyngeal colostrum leads to sustained breast milk feedings in preterm infants. Pediatr Neonatol. 2017; 58:534-40

10. Rodriguez NA, Meier PP, Groer MW, Zeller JM, Engstrom JL, Fogg L. A pilot study to determine the safety and feasibility of oropharyngeal administration of own mother's colostrum to extremely low-birth-weight infants. Adv Neonatal Care. 2010; 10:206-12.

11. Sohn K, Kalanetra KM, Underwood MA. Buccal administration of human colostrum: impact on the oral microbiota of premature infants. J Perinatol. 2016;36:106-11.

12. Tannock GW. Identification of lactobacilli and bifidobacteria. Curr Issues Mol Biol. 1999;1:53-64.

13. Tuomisto $\mathrm{H}$. A consistent terminology for quantifying species diversity? Yes, it does exist. Oecologia. 2010;164:853-60.

14. Schanler RJ, Goldblum RM, Garza C, Goldman AS. Enhanced fecal excretion of selected immune factors in very low birth weight infants fed fortified human milk. Pediatr Res. 1986;20: 711-5.

15. Spiegler J, Preuß M, Gebauer C, Bendiks M, Herting E, Göpel W, German Neonatal Network (GNN). Does breastmilk influence the development of bronchopulmonary dysplasia? J Pediatr. 2016;169:76-80. 